\title{
Sonographic Evaluation of Lisfranc Ligament Injuries
}

\author{
Suzanne Woodward, MD, Jon A. Jacobson, MD, \\ John E. Femino, MD, Yoav Morag, MD, David P. Fessell, MD, \\ Qian Dong, MD
}

\begin{abstract}
Objective. This study characterized the sonographic appearances of Lisfranc injuries. Methods. Sonography reports (2000-2007) were searched for "Lisfranc," resulting in 10 patients. Sonographic images of affected and asymptomatic contralateral feet were reviewed, recording the thickness of the dorsal ligament between the first (medial) cuneiform (C1) and second metatarsal (M2) ligaments, distance between $\mathrm{C} 1$ and $\mathrm{M} 2$, and change in this distance with weight bearing, hyperemia, and fractures. Correlations were made to clinical, surgical, and other imaging findings. Results. In 5 asymptomatic feet, the dorsal C1-M2 ligament was 0.9 to $1.2 \mathrm{~mm}$ thick, and the C1-M2 distance was 0.5 to $1 \mathrm{~mm}$. Of the symptomatic feet, 1 group $(n=3)$ had normal sonographic findings (thickness, 0.9-1.1 mm; distance, 0.6-0.7 mm; all had normal radiographic findings and follow-up, and 1 had normal magnetic resonance imaging $[\mathrm{MRI}]$ findings). Another group $(n=3)$ had abnormal hypoechogenicity and thickening of the dorsal C1-M2 ligament (1.4-2.3 mm), a normal C1-M2 distance $(0.6-0.7 \mathrm{~mm})$, and no widening with weight bearing (1 of 1), consistent with a ligament sprain (1 had normal computed tomographic [CT] findings, and all had uneventful follow-up). The third group $(n=4)$ had nonvisualization of the dorsal C1-M2 ligament, an increased C1-M2 distance of 2.5 to $3.1 \mathrm{~mm}$, and further widening with weight bearing (3 of 4 ) from Lisfranc ligament disruption (shown at surgery in 2, MRI in 1, and CT in 1). Conclusions. Nonvisualization of the dorsal C1-M2 ligament and a C1-M2 distance of $2.5 \mathrm{~mm}$ or greater were indirect signs of a Lisfranc ligament tear. Dynamic evaluation with weight bearing showed widening of the space between C1 and M2. Key words: foot; ligament; sonography; trauma.
\end{abstract}

Abbreviations

C1, first cuneiform; CT, computed tomography; MRI, magnetic resonance imaging; $\mathrm{M} 2$, second metatarsal
Received October 6, 2008, from the Department of Radiology, University of Michigan, Ann Arbor, Michigan USA (S.W., J.A.J., Y.M., D.P.F., Q.D.); and Department of Orthopedic Surgery, University of lowa, lowa City, Iowa USA (J.E.F.). Revision requested October 17, 2008. Revised manuscript accepted for publication October 21, 2008.

Address correspondence to Jon A. Jacobson, MD, Department of Radiology, University of Michigan, 1500 E Medical Center Dr, Ann Arbor, MI 48109-0326 USA.

E-mail: jjacobsn@umich.edu

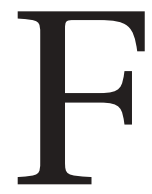

racture dislocations of the tarsometatarsal joints are often referred to as Lisfranc injuries. Named after the French surgeon who performed amputations through these joints, Lisfranc injuries have been associated with many different injuries to the tarsometatarsal joint complex. The Lisfranc ligament, which extends obliquely between the lateral aspect of the first (medial) cuneiform $(\mathrm{Cl})$ ligament to the medial aspect of the second metatarsal (M2) ligament base, is of paramount importance to the stabilization of the tarsometatarsal joints. ${ }^{1}$ Complications of Lisfranc ligament injuries can be devastating and may include malreduction resulting in posttraumatic osteoarthrosis and chronic foot pain. ${ }^{2,3}$

Accurate diagnosis of Lisfranc injuries may be difficult, and a delayed diagnosis or misdiagnosis occurs in up to $20 \%$ of cases. ${ }^{4}$ Evaluation typically begins with conventional radiographs of the foot. However, because of overlapping osseous structures on routine radiographic views, 
fractures can easily be missed. ${ }^{5}$ Furthermore, in cases in which no malalignment is visible on conventional radiographs, ligamentous injuries will be overlooked. Stress radiographs may be helpful but may still be erroneously reported as normal. ${ }^{6}$ Computed tomography (CT) or magnetic resonance imaging (MRI) is often performed in inconclusive cases. ${ }^{3,5,6}$

Sonography of the foot, including dynamic assessment, is one imaging method that can be performed for unexplained foot pain. At our institution, we have identified Lisfranc joint injuries on sonography, although there is no report of such an evaluation in the literature to our knowledge. The purpose of this study was to retrospectively characterize the sonographic appearances of Lisfranc injuries of the foot.

\section{Materials and Methods}

Institutional Review Board approval was obtained, and informed consent was waived. Sonography reports in the radiology information system were searched from January 1999 through September 2007 for the key word "Lisfranc." Cases were excluded if imaging was not available. The search yielded 10 patients. Sonography of the foot in each patient was ordered as part of the clinical care of the patient. The sonographic examinations were completed by 1 of 5 musculoskeletal radiologists (2-13 years of experience) using 7- to $14-\mathrm{MHz}$ linear transducers (iU22 and HDI 5000; Philips Healthcare, Bothell, WA; and LOGIQ 9; GE Healthcare, Milwaukee, WI). Images were acquired and saved in either digital or hard copy format.

With regard to the ultrasound scanning technique, the tarsometatarsal joints were imaged in the coronal and sagittal planes. The C1-M2 interval is located with coronal scanning initially over the first and second metatarsals (Figure 1). As the transducer is moved posteriorly, the first tarsometatarsal joint is visualized. Continuing to scan in a posterior direction will then bring the dorsal surface of $\mathrm{Cl}$ into view with the adjacent M2 base. In contrast to the rounded shape of the M2 dorsal surface, the $\mathrm{C} 1$ dorsal surface is more angulated. The dorsal C1-M2 ligament will appear hyperechoic and fibrillar, with a characteristic notch in $\mathrm{Cl}$ at its attachment (Figure 1).
Figure 1. Normal C1-M2 articulation in a 42-year-old man. A, The sonographic evaluation begins in the coronal plane transverse to the first metatarsal (M1) ligament and M2. B, The ultrasound transducer is moved posteriorly across the first tarsometatarsal joint and is positioned in the coronal plane transverse to $\mathrm{C} 1$ and M2. Note the normal dorsal C1-M2 ligament (arrows) and characteristic bone contour or plateau of $\mathrm{C} 1$ at the ligament attachment (arrowhead). C, A skeletal model shows the two transducer positions (white lines) as described in A and B; yellow indicates the location of the Lisfranc and dorsal C1-M2 ligaments.

\section{A}

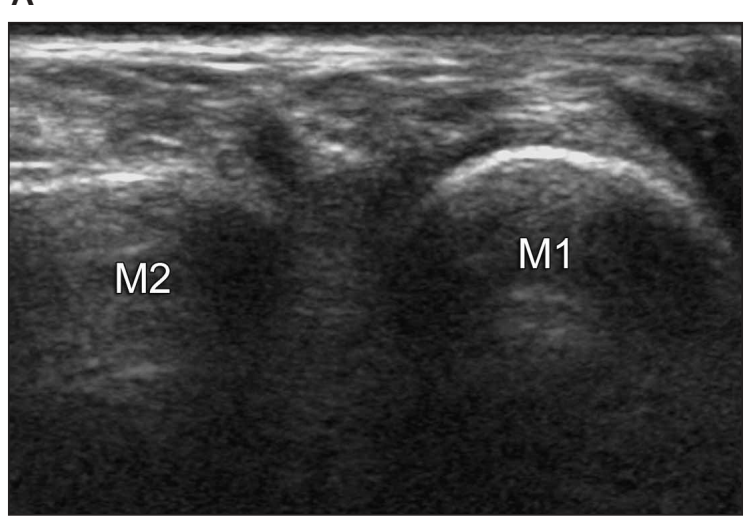

B

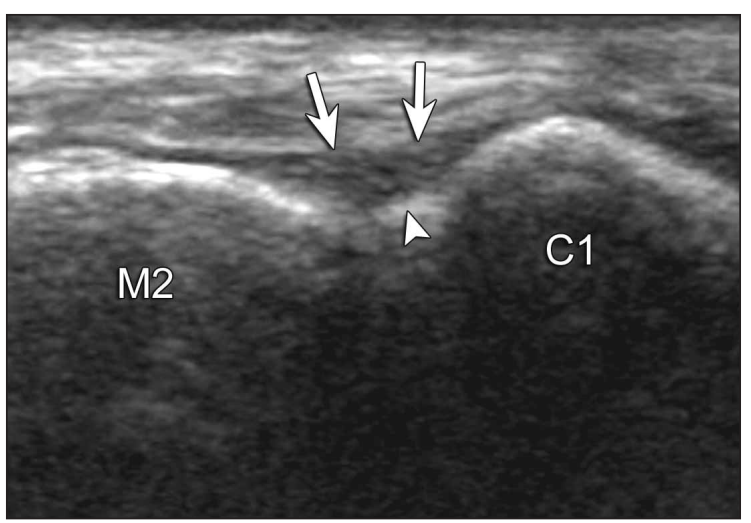

C

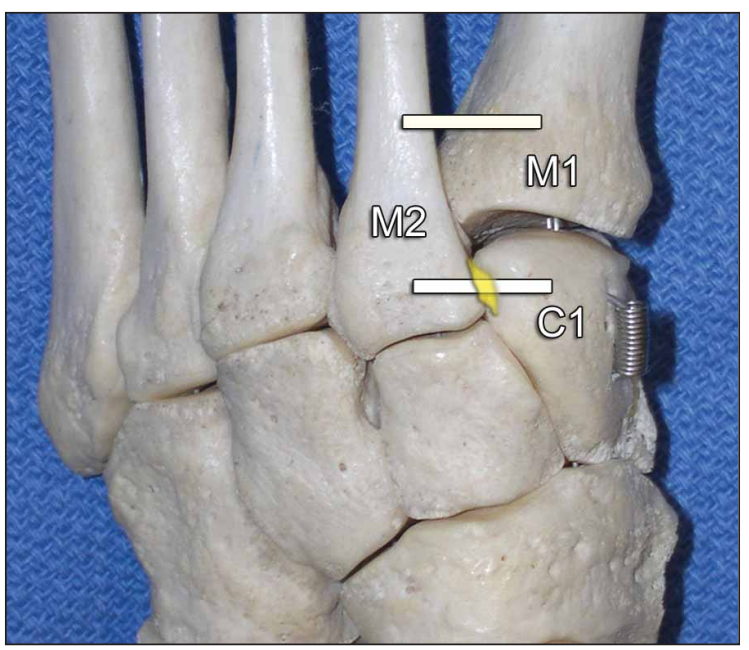


Measurements are taken at this site. Depending on the amount of adjacent hyperechoic fat, the gain setting of the ultrasound machine and the angle between the ligament and ultrasound beam, the normal ligament may appear mildly hypoechoic, although the superficial border will not be convex, and a normal fibrillar echo texture will be seen.

Sonographic images and the original sonographic reports were retrospectively reviewed by a radiology resident (third year of training) and fellowship-trained musculoskeletal radiologist (13 years of experience) by consensus. Recorded data included the thickness of the dorsal ligament between $\mathrm{C} 1$ and $\mathrm{M} 2$, the space (millimeters) between $\mathrm{C} 1$ and $\mathrm{M} 2$, any change in this distance with weight bearing when applicable, the presence of hyperemia on color or power Doppler imaging, and the presence of a fracture (cortical step-off deformity). Data were recorded from the symptomatic extremity as well as the contralateral asymptomatic extremity when obtained. The medical records were subsequently reviewed to correlate sonographic findings with surgical reports, clinical notes, radiography, CT, and MRI, where available.

\section{Results}

The 10 patients were on average 36 years of age (range, 11-63 years) and included 5 women and 5 men. Of the symptomatic feet, the right side was affected in 4 and the left in 6 . In 5 of the patients, the asymptomatic foot was scanned and used for comparison. Of the 10 patients, all had routine foot radiographs (9 were weight bearing); 3 had CT examinations; 3 had MRI examinations; and 2 had surgery.

In the 5 asymptomatic feet, the dorsal C1-M2 ligament was hyperechoic with a thickness of 0.9 to $1.2 \mathrm{~mm}$, and the C1-M2 distance was 0.5 to 1 $\mathrm{mm}$ (Figure 2). Given these findings, the symptomatic feet could be divided into 3 groups. One group $(n=3)$ had similar findings (hyperechoic ligament, thickness of 0.9-1.1 mm, and C1-M2 distance of $0.6-0.7 \mathrm{~mm}$ ); all had normal clinical follow-up and radiographic findings, and 1 had normal MRI findings. Another group $(n=3)$ had hypoechogenicity and thickening of the dorsal C1-M2 ligament (1.4-2.3 mm) with a convex

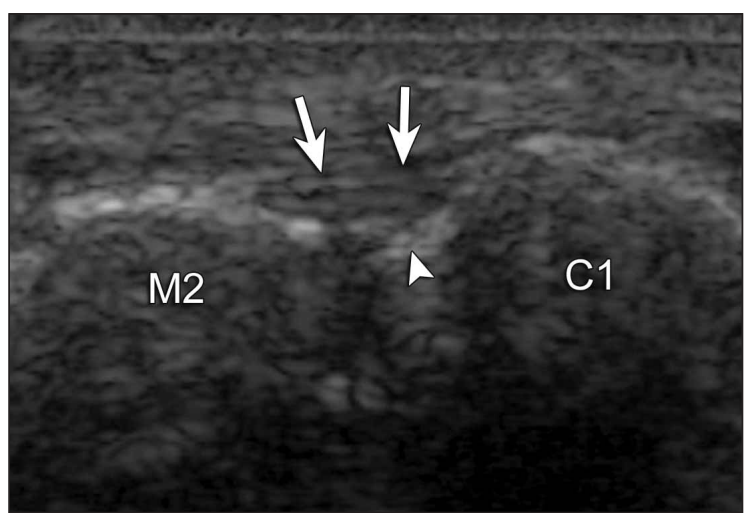

Figure 2. Normal C1-M2 articulation (asymptomatic foot) in a 40-year-old woman. Sonography in the coronal plane transverse to the metatarsal shafts shows a normal dorsal C1-M2 ligament (arrows; thickness measured as $1 \mathrm{~mm}$ ). Note the characteristic bone contour of $\mathrm{C} 1$ at the ligament attachment (arrowhead). The C1-M2 space measured $0.7 \mathrm{~mm}$.

superficial border (Figure 3), a C1-M2 distance of 0.6 to $0.7 \mathrm{~mm}$, and no widening with weight bearing (1 of 1); 1 had negative CT findings; and all 3 had no malalignment on radiography and uneventful clinical follow-up. The third group $(n=4)$ had findings consisting of hypoechogenicity and nonvisualization of the dorsal C1-M2 ligament, a C1-M2 distance of 2.5 to $3.1 \mathrm{~mm}$, and further widening with weight bearing ( 3 of 4; Figures 4 and 5); Lisfranc ligament disruption was confirmed at surgery in 2, MRI in 1 , and CT in 1.

Figure 3. Dorsal C1-M2 ligament sprain in a 41-year-old man. Sonography in the coronal plane transverse to the metatarsal shafts shows a hypoechoic and swollen dorsal C1-M2 ligament (arrows; thickness measured as $2.3 \mathrm{~mm}$ ). Note the normal C1M2 space measuring $0.6 \mathrm{~mm}$ (arrowhead).

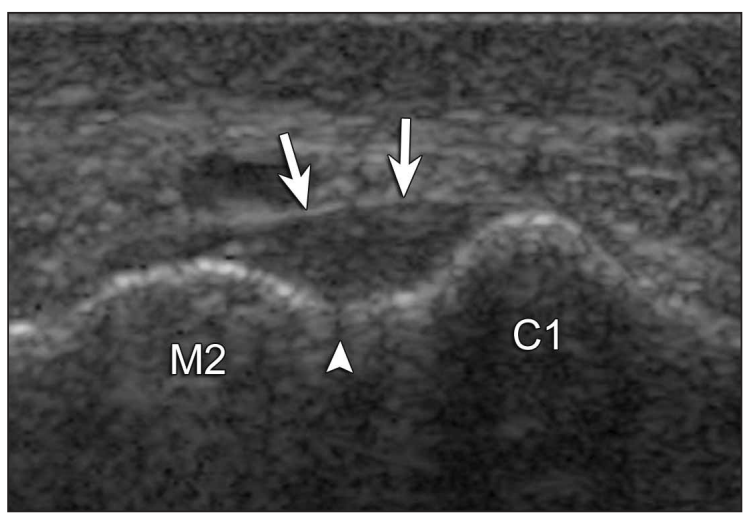


Four of the 10 subjects had color or power Doppler imaging. Of these, 2 showed increased flow dorsally between $\mathrm{C} 1$ and M2 (Figure 4C; both had Lisfranc ligament tears), and 2 did not show any flow (1 had a Lisfranc ligament tear, and the other had a sprain). With regard to fractures, 1 of 10 patients showed a fracture on sonography, confirmed on radiography and MRI.

\section{Discussion}

Prompt diagnosis of a Lisfranc joint injury is important to allow appropriate treatment and avoid long-term disability. This study shows that there are characteristic sonographic findings that indicate a Lisfranc ligament tear. Although visualization of the Lisfranc ligament proper is not possible with sonography because of the narrow space between $\mathrm{Cl}$ and $\mathrm{M} 2$, assessment of the dorsal C1-M2 ligament and forefoot alignment can indicate a Lisfranc ligament injury. Dynamic imaging in the evaluation of alignment offers additional information.

The tarsometatarsal joint complex (Lisfranc joint) articulates the distal tarsal bones with the metatarsal bases. The stabilization of the joint is complex and consists of a dorsal ligament system, plantar ligaments, and interosseous liga-

Figure 4. Lisfranc ligament tear (diagnosed at CT) in a 25-year-old man. A, Sonography in the coronal plane transverse to the metatarsal shafts shows abnormal hypoechogenicity, absence of the dorsal C1-M2 ligament (arrow), and widening between C1 and M2 (brace) measuring $3.1 \mathrm{~mm}$. B, Sonography in the coronal plane transverse to the metatarsal shafts with patient weight bearing shows findings similar to those in A (arrow) but with increased widening between C1 and M2 (brace). C, Color Doppler imaging shows increased blood flow dorsally between $\mathrm{C} 1$ and M2. D, Non-contrast-enhanced axial CT (0.5-mm slice thickness) shows malalignment between the second cuneiform (C2) ligament and M2 (arrow) and widening between C1 and M2.

A

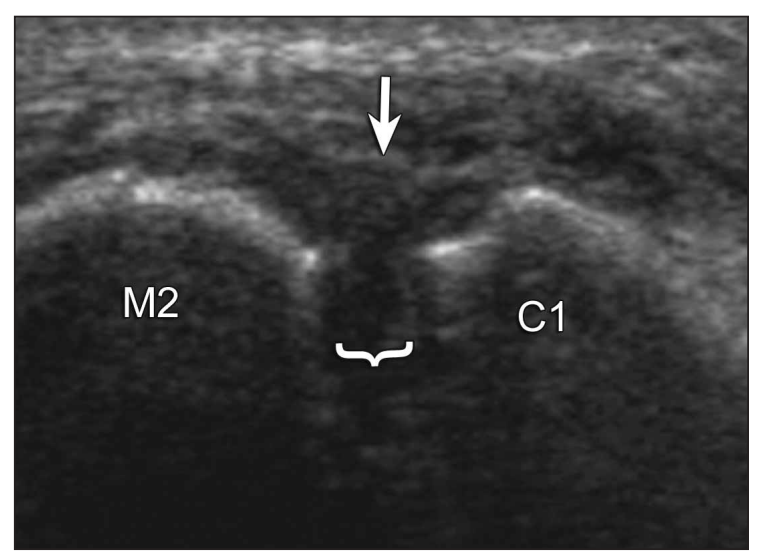

C

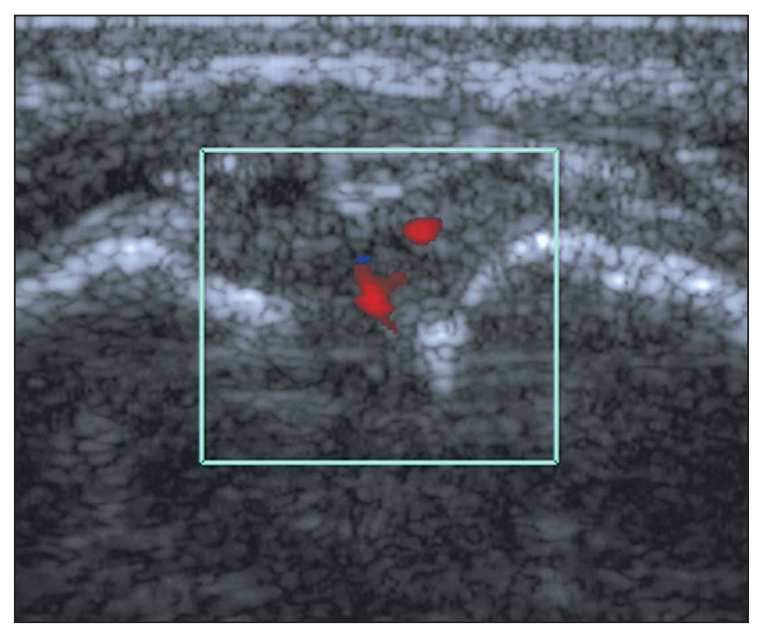

B

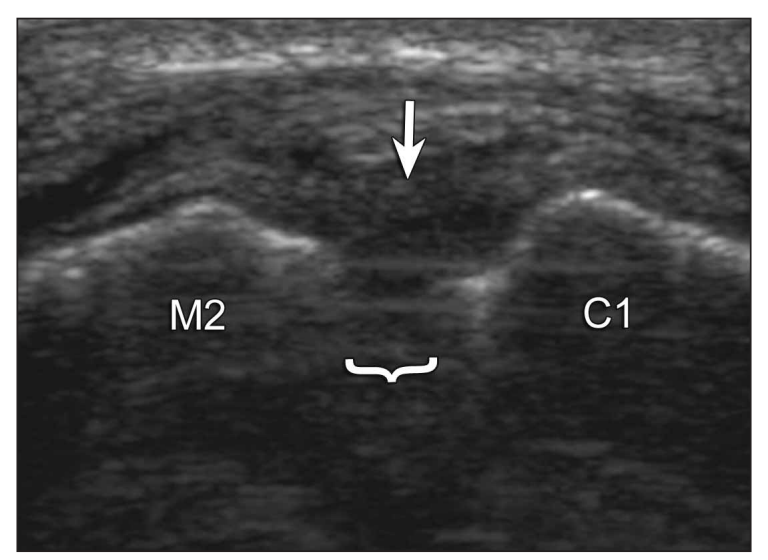

D

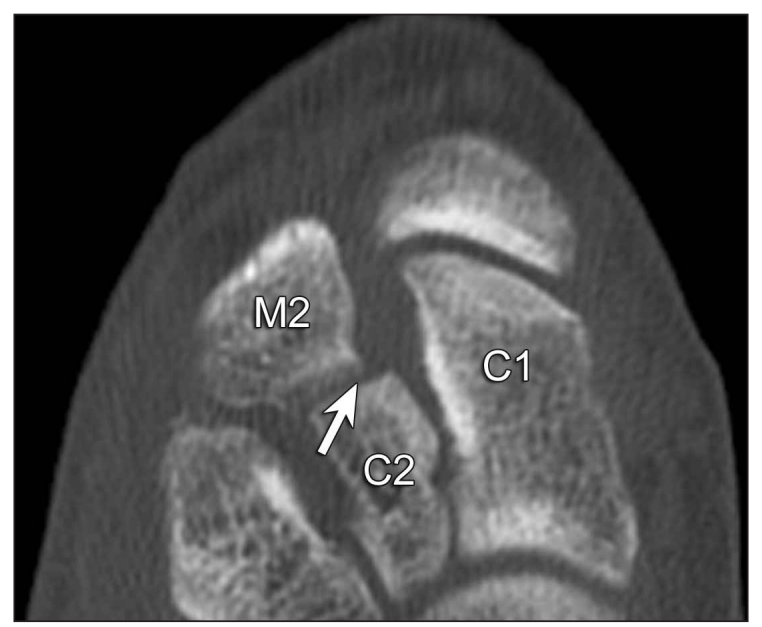


Figure 5. Lisfranc ligament tear (diagnosed at surgery) in a 50year-old man. A, Sonography in the coronal plane transverse to the metatarsal shafts shows abnormal hypoechogenicity, absence of the dorsal C1-M2 ligament (arrow), and widening between $\mathrm{C} 1$ and $\mathrm{M} 2$ (bracket) measuring $2.5 \mathrm{~mm}$. B, Sonography in the coronal plane transverse to the metatarsal shafts with patient weight bearing shows findings similar to those in $\mathrm{A}$ (arrow) but with increased widening between $\mathrm{C} 1$ and M2 (brace). C, Non-contrast-enhanced axial CT (0.5-mm slice thickness) shows equivocal malalignment between $\mathrm{C} 2$ and $\mathrm{M} 2$ (arrow) and equivocal widening between $\mathrm{C} 1$ and $\mathrm{M} 2$.

\section{A}

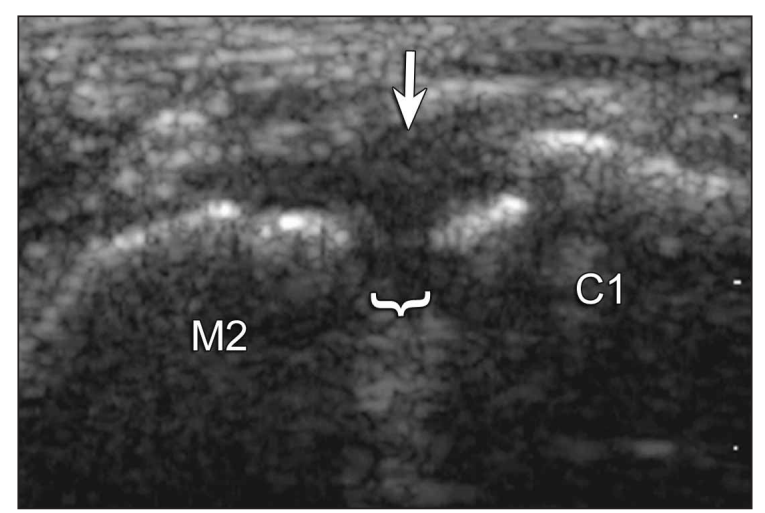

B

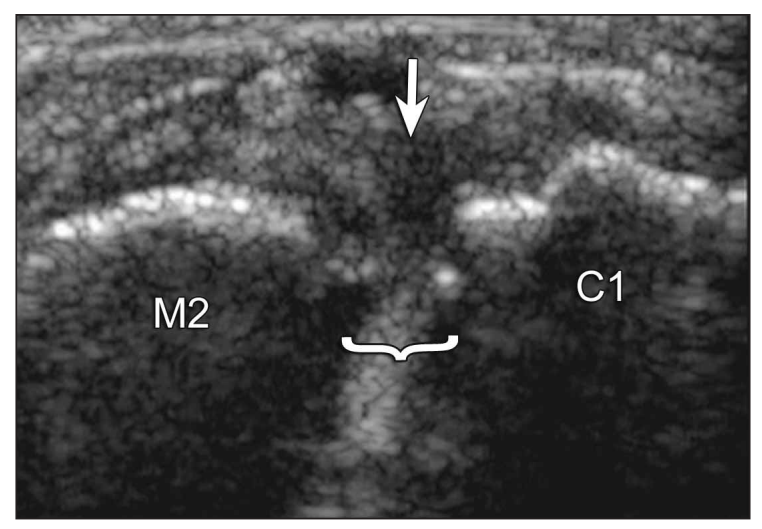

c

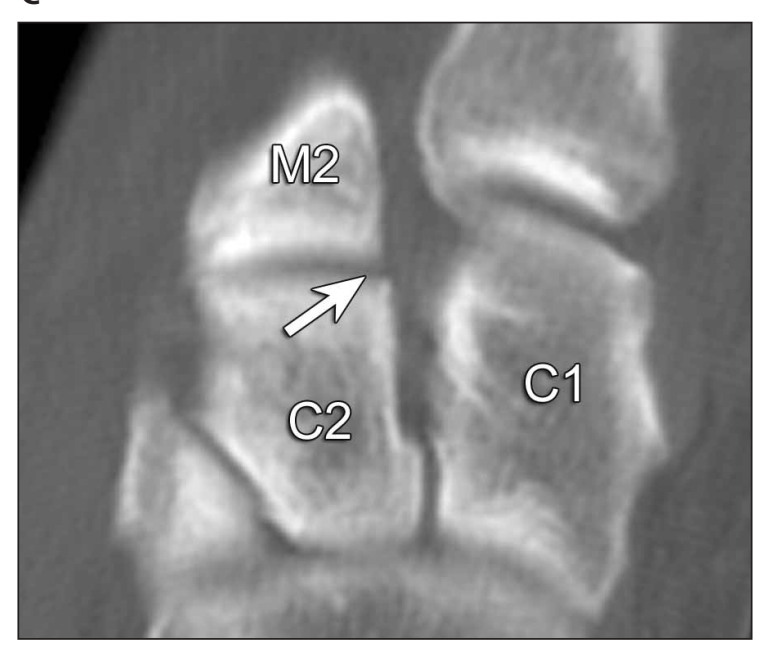

ments. ${ }^{7,8}$ The dorsal ligament system represents thin, flat transverse, oblique, and longitudinal bands that connect tarsal bones to tarsal bones, tarsal bones to metatarsals, and metatarsals to metatarsals, including $\mathrm{C} 1$ to $\mathrm{M} 2{ }^{7}$ The plantar ligaments similarly consist of transverse and longitudinal ligaments that interconnect the osseous structures, including $\mathrm{C} 1$ to $\mathrm{M} 2 .^{7}$ Among the interosseous ligaments is the Lisfranc ligament proper, the largest ligament of the tarsometatarsal joints, arising from the lateral aspect of $\mathrm{C} 1$ extending distal, lateral, and plantar to attach to the medial aspect of M2. ${ }^{7}$ Of note, there is no mechanically significant ligamentous connection between the first and second metatarsal bases, but instead, the Lisfranc ligament provides stability at this articulation. $^{7}$

The Lisfranc joint may be injured with plantar flexion and forced pronation or supination. ${ }^{5}$ This can result in ligament disruption, a fracture, and possible malalignment. When a patient has midfoot pain and swelling after trauma, it is important to exclude a fracture and consider a ligamentous injury. When the Lisfranc ligament is disrupted, dislocation most often occurs dorsally, laterally, or dorsolaterally because the M2 base is wider dorsally than ventrally. ${ }^{9}$

The evaluation for a Lisfranc joint injury typically begins with conventional radiography. However, radiographs may show false-negative findings, and multiple studies have shown conventional radiographs to be unreliable for detection of Lisfranc dislocation, particularly for detection of subtle injuries or ligamentous injuries without associated fractures. ${ }^{5}$ Although a distance of $2 \mathrm{~mm}$ or greater between $\mathrm{Cl}$ and M2 has been shown to correlate with a complete tear of the Lisfranc ligament, a Lisfranc ligament injury may be associated with distances of less than this. ${ }^{10}$ In addition, it has been shown that malalignment of 1 or $2 \mathrm{~mm}$ is not reliably identified on radiography. ${ }^{9}$ Weight-bearing radiographs may be helpful to show malalignment but are often limited by patient pain and remain unreliable. ${ }^{5}$ In a cadaveric model, manual stress radiographs of the foot have been shown to depict more displacement than weight-bearing radiography alone. ${ }^{11}$ Several studies have also emphasized the importance of CT in the evaluation for fractures and bony alignment when con- 
ventional radiographs are unrevealing and clinical suspicion remains high., ${ }^{5,69}$ Magnetic resonance imaging has been used to show fractures, bony alignment, and the integrity of the Lisfranc ligament. ${ }^{2,5,8,10}$ The results of this study suggest a potential role for sonography as well.

When performing sonography of the midfoot, evaluation of the dorsal C1-M2 ligament is important because it can be an indirect sign of a Lisfranc ligament tear. The normal dorsal C1-M2 ligament, which appears hyperechoic with a thickness of 0.9 to $1.2 \mathrm{~mm}$, is easily identified given its superficial location and adjacent distinctive osseous landmarks (Figure 1). Of note, a normal dorsal C1-M2 ligament may appear mildly hypoechoic depending on the amount of adjacent hyperechoic fat, the gain setting of the ultrasound machine, and whether the ultrasound beam is perpendicular to the ligament (Figure 1); regardless, a fibrillar echo texture without a convex superficial margin should be seen with a normal dorsal C1-M2 ligament. In this study, the 4 cases of Lisfranc ligament disruption had associated disruption of the dorsal C1-M2 ligament (Figures 4 and 5). In addition, a Lisfranc ligament tear was excluded in every case in which the dorsal C1-M2 ligament was normal on sonography. This may be explained by the relatively small size of the dorsal C1-M2 ligament compared with the Lisfranc ligament. Evaluation of a larger population with midfoot injuries would determine whether these associations are consistent. In 3 cases, the dorsal C1-M2 ligament was intact but hypoechoic and swollen (1.4- to 2.3-mm thickness; Figure 3); imaging and follow-up did not show malalignment, and this was interpreted as a dorsal C1-M2 ligament sprain.

In addition to disruption of the dorsal C1-M2 ligament, widening of the space between $\mathrm{Cl}$ and M2 is another important and probably more specific indirect sign of a Lisfranc ligament tear. Normally this space between cortical surfaces of $\mathrm{C} 1$ and $\mathrm{M} 2$ measures 0.5 to $1.0 \mathrm{~mm}$ in the coronal plane on sonography. With a Lisfranc ligament tear, this space measured $2.5 \mathrm{~mm}$ or greater (Figures 4 and 5). Although a widened C1-M2 space in the setting of a Lisfranc ligament tear was evident on sonography with the patients supine, this space further increased with weight bearing (Figures 4 and 5). The dynamic imaging of sonography is a potential benefit because imaging during weight bearing may increase sensitivity in the diagnosis of a Lisfranc ligament tear.

Other sonographic findings associated with Lisfranc ligament tears were more variable. Increased blood flow dorsally between $\mathrm{C} 1$ and M2 was shown in 2 of the 3 cases of Lisfranc ligament tears in which color or power Doppler imaging was performed (Figure 4C). A cortical step-off representing a fracture was seen in 1 of 4 cases with Lisfranc ligament tears. Fracture assessment in the midfoot region with sonography is limited because of the complex anatomy. It is also important not to misinterpret an os intermetatarsus as a fracture fragment.

The limitations of our study included its retrospective nature and resulting small number of patients included. A larger prospective study would be needed to further validate these results. In addition, not all patients had imaging or surgical proof, although a correlation was made to the clinical outcome in such cases.

In conclusion, absence of the dorsal C1-M2 ligament, a C1-M2 interval of $2.5 \mathrm{~mm}$ or more, and progressive widening on a dynamic sonographic evaluation are indirect signs of Lisfranc ligament tears. Sonography can play a role in the assessment of forefoot and midfoot pain, and familiarity with these findings is important when performing sonography of the foot.

\section{References}

1. Sarrafian SK. Anatomy of the Foot and Ankle: Descriptive, Topographic, Functional. Philadelphia, PA: JB Lippincott Co; 1993.

2. Preidler KW, Brossmann J, Daenen B, Goodwin D, Schweitzer M, Resnick D. MR imaging of the tarsometatarsal joint: analysis of injuries in 11 patients. AJR Am J Roentgenol 1996; 167:1217-1222.

3. Talarico RH, Hamilton GA, Ford LA, Rush SM. Fracture dislocations of the tarsometatarsal joints: analysis of interrater reliability in using the modified Hardcastle classification system. J Foot Ankle Surg 2006; 45:300-303.

4. Hardcastle PH, Reschauer R, Kutscha-Lissberg E, Schoffmann W. Injuries to the tarsometatarsal joint: incidence, classification and treatment. J Bone Joint Surg $\mathrm{Br}$ 1982; 64:349-356.

5. Preidler KW, Peicha G, Lajtai G, et al. Conventional radiography, CT, and MR imaging in patients with hyperflexion injuries of the foot: diagnostic accuracy in the detection of bony and ligamentous changes. AJR Am J Roentgenol 1999; 173:1673-1677. 
6. Goiney RC, Connell DG, Nichols DM. CT evaluation of tarsometatarsal fracture-dislocation injuries. AJR Am J Roentgenol 1985; 144:985-990.

7. de Palma L, Santucci A, Sabetta SP, Rapali S. Anatomy of the Lisfranc joint complex. Foot Ankle Int 1997; 18:356-364.

8. Preidler KW, Wang YC, Brossmann J, Trudell D, Daenen B, Resnick D. Tarsometatarsal joint: anatomic details on MR images. Radiology 1996; 199:733-736.

9. Lu J, Ebraheim NA, Skie M, Porshinsky B, Yeasting RA. Radiographic and computed tomographic evaluation of Lisfranc dislocation: a cadaver study. Foot Ankle Int 1997; 18:351-355.

10. Potter HG, Deland JT, Gusmer PB, Carson E, Warren RF. Magnetic resonance imaging of the Lisfranc ligament of the foot. Foot Ankle Int 1998; 19:438-446.

11. Kaar S, Femino J, Morag Y. Lisfranc joint displacement following sequential ligament sectioning. J Bone Joint Surg Am 2007; 89:2225-2232. 